\title{
Blockade of nicotine sensitization by methanol extracts of Glycyrrhizae radix mediated via antagonism of accumbal oxidative stress
}

\author{
Zheng Lin Zhao ${ }^{1 \dagger}$, Sang Chan Kim² ${ }^{2 \dagger}$, Hong Feng Liư ${ }^{3}$, Yi Yan Wu ${ }^{3}$, Li Bo Li ${ }^{1}$, Yu Hua Wang ${ }^{1}$, Yu Jiao ${ }^{1}$, Yu Fan ${ }^{1,2}$,
} Chul Won Lee ${ }^{2}$, Bong Hyeo Lee ${ }^{2}$,I Je Cho ${ }^{2}$, Chae Ha Yang ${ }^{2}$ and Rong Jie Zhao ${ }^{1,2^{*}}$

\begin{abstract}
Background: We previously reported that a methanol extract of Glycyrrhizae radix (MEGR) blocked methamphetamineinduced locomotor sensitization and conditioned place preference in rats. In the present study, the effects of MEGR on repeated nicotine-induced locomotor sensitization and enhanced extracellular dopamine (DA) release in the nucleus accumbens (Nacc) were evaluated.

Methods: Male Sprague-Dawley rats received repeated administrations of nicotine ( $0.4 \mathrm{mg} / \mathrm{kg}$, subcutaneous) or saline twice a day for $7 \mathrm{~d}$ and were challenged with nicotine $4 \mathrm{~d}$ after the last daily dosing. During the 4-d withdrawal period, the rats were treated once a day with MEGR (60 or $180 \mathrm{mg} / \mathrm{kg} / \mathrm{d}$ ). Extracellular DA levels were measured by in vivo microdialysis, the malondialdehyde levels and the activities of superoxide dismutase and catalase in the Nacc were biochemically evaluated, and the expression of antioxidant proteins was confirmed by Western blot assays. All data were assessed with analysis of variance tests followed by post-hoc comparison tests and $p$ values $<0.05$ were considered statistically significant.

Results: The expression of repeated nicotine-induced locomotor sensitization was dose-dependently attenuated by MEGR, and $180 \mathrm{mg} / \mathrm{kg} / \mathrm{d}$ MEGR significantly inhibited augmented accumbal DA release induced by a direct local challenge of nicotine. Moreover, $180 \mathrm{mg} / \mathrm{kg} / \mathrm{d}$ MEGR reversed increases in malondialdehyde production, decreases in superoxide dismutase and catalase activities, and the reduced expression of nuclear factor erythroid 2-related factor 2 and heme oxygenase 1 in the nicotine-sensitized Nacc.
\end{abstract}

Conclusions: These results suggest that MEGR inhibited nicotine-induced locomotion and dopaminergic sensitization via antioxidant action.

Keywords: Nicotine, Sensitization, Glycyrrhizae radix, Dopamine, Nucleus accumbens, Oxidative stress

\section{Background}

It is now known that cigarette smoking can greatly endanger public health worldwide and that nicotine dependence is likely the prime culprit associated with the failure to quit smoking [1]. However, other than nicotine

\footnotetext{
* Correspondence: zhao_rongjie@yahoo.com

${ }^{\dagger}$ Equal contributors

'School of Mental Health, Qiqihar Medical University, 333 Bukuibei Street, Jianhua District, Qiqihar 161006, China

${ }^{2}$ College of Korean Medicine, Daegu Haany University, Gyeongsan 38610, Republic of Korea

Full list of author information is available at the end of the article
}

replacement therapy, which has limited effectiveness, there are no available pharmacotherapies that effectively aid quitting [2]. Although nicotine has a relatively lower potency than most opiates and psychostimulants, its rewarding effect is a key motivational factor that sustains nicotine dependence [3]. In rodent studies, the rewarding effects of nicotine typically manifest as behavioral sensitization such as enhancement in locomotor activity that occurs after repeated nicotine treatment (RNT) [4]. Additionally, this sensitization is positively associated 
with other addiction-related behaviors, such as nicotine seeking and self-administration.

Similar to other addictive drugs, nicotine induces increased levels of accumbal dopamine (DA) release; these changes are neurochemically responsible for the rewarding effects of the drug [5]. Furthermore, RNT leads to a sensitized dopaminergic state such that a challenge dose of nicotine in a subject pre-exposed to nicotine produces a significant augmentation in accumbal DA release compared with a subject naive to nicotine [6]. This process is thought to be the neurobiological underpinning of behavioral sensitization.

To date, the exact biochemical mechanisms underlying the sensitization of the dopaminergic response after repeated treatment with drugs have yet to be fully elucidated. However, an increasing amount of evidence points to the critical role of reactive oxygen species (ROS) in this regard [7]. There are increased ROS levels in brain regions related to reward after repeated exposure to a variety of abused drugs, including nicotine [8]. Additionally, TEMPOL, which is a ROS scavenger, attenuates the promotion of cocaine and methamphetamine self-administration by preventing the sensitization of DA release in the nucleus accumbens (Nacc) $[9,10]$. Further, abolishment of oxidative stress in the Nacc and prefrontal cortex can block repeated cocaine-induced rat locomotor sensitization [11]. Therefore, it is possible that the increase in accumbal oxidative stress induced by repeated administration of drugs of abuse might be a promising target for the treatment of addiction.

The radix of Glycyrrhizae uralensis [Glycyrrhizae radix (G. radix)] has traditionally been used in Oriental medicine for detoxification, the treatment of various injuries, and swelling, due to its ability to replenish and invigorate deficiencies in "Qi and Blood" [12]. G. radix contains flavonoids and different types of pentacyclic triterpene saponin, a group which includes liquiritigenin, liquiritin, isoliquiritigenin, glycyrrhizin, and glycyrrhizic acid [13]. Historically, G. radix has received pharmacological interest due to its anti-oxidant, anti-inflammatory, antitumor, and antitussive actions $[14,15]$. However, over the past decade, several studies have revealed G. radix has neuropharmacological properties such as neuroprotective, antidepressant, and anxiolytic effects [16-18]. In previous studies, we found extracts from $G$. radix suppressed acute cocaine- and methamphetamine- induced increases in accumbal DA levels [19] and prevented acute methamphetamine-induced hyperlocomotion in rats [20], indicating inhibitory effects of G. radix against pharmacological actions of psychostimulants. Moreover, in other studies, we also demonstrated G. radix improved the behavioral and neurochemical abnormalities caused by repeated psychostimulant treatment such that extracts from G. radix attenuated methamphetamine- induced locomotor sensitization and conditioned place preference [21] and isoliquiritigenin ameliorated repeated methamphetamine-induced loss of striatal dopamine transporter densities and tyrosine hydroxylase activities [22]. Since nicotine belongs to the category of psychostimulants and shares common neurochemical and behavioral traits with the typical psychostimulants when given repeatedly [23], the results of these studies collectively raise the possibility that $G$. radix can prevent the occurrence of aberrant neurochemical and behavioral changes by RNT.

In the meantime, in the above mentioned studies, we have shown that $G$. radix exerts its neuropharmacological actions via glutamatergic and gamma-aminobutyric acid (GABA)ergic receptors [19, 21], which is also supported by the study done by others [24]. However, since the primary pharmacological property of $G$. radix is its antioxidant capabilities, and as reported by Zeng et al. that isoliquiritigenin alleviated intracerebral hemorrhageinduced brain injury in rats via suppressing excessive ROS production [25], it is possible that the neuroprotective effects of $G$. radix against repeated psychostimulant treatment are mediated, at least in part, via its antioxidant activities.

Therefore, in the present study, we aimed to evaluate the potential therapeutic effects of $G$. radix on RNT-induced locomotor and accumbal dopaminergic sensitization in rats and to further determine whether these effects are associated with its antioxidant actions.

\section{Methods}

\section{Preparation of an extract of $G$. Radix}

The methanol extract from G. radix (MEGR) was prepared as previously described [21]. Briefly, G. radix was obtained from Dae-Won pharmacy (Daegu, Republic of Korea), identified by Professor Sang Chan Kim (College of Oriental Medicine, Daegu Haany University, Daegu, Republic of Korea) and ground into fine powder. The powdered $G$. radix was extracted in methanol for $48 \mathrm{~h}$, filtered through a $0.22-\mu \mathrm{m}$ filter (Nalgene; NY, USA), and then lyophilized in a vacuum evaporator. The amount of MEGR was estimated based on the dry weight of the lyophilized MEGR; the yield was $18.36 \%$. Analysis with high-performance liquid chromatography (HPLC) has shown that MEGR contains glycyrrhizic acid, liquiritigenin, and isoliquiritigenin [21].

\section{Animals}

Male Sprague-Dawley rats were obtained from the Laboratory Animal Center at Qiqihar Medical University (Qiqihar, China). The rats were 9 weeks old (280-300 g) at the beginning of the experiments, and housed in Plexiglas cages (3 rats per cage), provided with ad libitum food and water, maintained in a filtered pathogen- 
free air environment at a temperature of $21-23{ }^{\circ} \mathrm{C}$ and $50 \%$ relative humidity, and on a 12:12 h light/dark cycle. All animal procedures were performed in accordance with the National Institutes of Health Guide for the Care and Use of Laboratory Animals and were approved by the Animal Care and Use Committee of Qiqihar Medical University (Approval Number: QMU-AECC-2015-26).

\section{Locomotor activity test}

Locomotor activity was assessed in a rectangular box $\left(40 \times 40 \times 45 \mathrm{~cm}^{3}\right)$ with a floor and walls that were made from clear Plexiglas painted black. The chamber was equipped with a video camera above the center of the floor, and all locomotor activity was monitored by a video tracking system using the Ethovision program (Noldus Information Technology BV, Wageningen, Netherlands).

\section{Nicotine-induced Locomotor sensitization and drug treatment}

Forty two rats were given subcutaneous (s.c.) administration of either saline or $0.4 \mathrm{mg} / \mathrm{kg}$ of (-)-nicotine hydrogen tartrate (Sigma, St. Louis, MO, USA) dissolved in saline ( $\mathrm{pH}$ 7.2; all doses expressed as free base) twice a day for 7 consecutive days in their home cages, randomly assigned to drug-treated groups (3 groups, $n=7$ ) and vehicle-treated groups (3 groups, $n=7$ ), and then underwent $4 \mathrm{~d}$ of withdrawal $[6,26]$. During the withdrawal period, the rats were orally treated with either distilled water (DW) or MEGR (60 or $180 \mathrm{mg} / \mathrm{kg} / \mathrm{d}$, dissolved in DW) once a day for $4 \mathrm{~d}$ $[20,21]$. Immediately after the final dose of DW or MEGR, the rats were adapted to the locomotor testing boxes for $60 \mathrm{~min}$ and then systemically challenged with either nicotine $(0.4 \mathrm{mg} / \mathrm{kg})$ or saline [26]. Following the challenge, the rats stayed in the boxes for an additional $60 \mathrm{~min}$ while locomotor activity was assessed (Fig. 1a). Immediately after the behavioral test, the rats were anesthetized with ether and decapitated, and the entire brain was removed and stored at $-80{ }^{\circ} \mathrm{C}$. Tissue samples from the Nacc shell (NaccSh; anterior-posterior: $1.7 \mathrm{~mm}$, medial-lateral: $0.8 \mathrm{~mm}$, and dorsal-ventral: $-7.4 \mathrm{~mm}$, based on the Paxinos and Watson rat brain atlas) [27] were punched from the stored brains for biochemical and Western blot analyses.

To investigate the effects of MEGR on sensitized accumbal DA release, another cohort of nicotine-withdrawn rats (submitted to the same treatment schedule described above) fitted with dialysis probes received a direct intraNaccSh infusion of nicotine $(3.0 \mathrm{mM})$ for $20 \mathrm{~min}$ [26] at $60 \mathrm{~min}$ after the fourth administration of MEGR. Subsequently, DA levels in the NaccSh were examined using in vivo microdialysis [26].

\section{Extracellular DA measurement}

A microdialysis probe guide cannula (CMA11, Carnegie Medicin, Stockholm, Sweden) was implanted into the
NaccSh using a stereotaxic instrument (Kopf Instruments, Tujunga, CA, USA) while the rats were under anesthesia (sodium pentobarbital, $50 \mathrm{mg} / \mathrm{kg}$, intraperitoneally). After the surgery, the rats were individually housed, and antibiotic treatment (bacitracin ointment and penicillin) and acetaminophen were applied for 3 days to minimize infection and pain in the rats. Following $7 \mathrm{~d}$ of post-surgery recovery, the rats were subjected to the nicotine sensitization schedule. On the fourth day after the last daily injection, microdialysis probes (CMA11, cuprophane dialysis membrane, $6000 \mathrm{Da}, 2-\mathrm{mm}$ length) were inserted into the NaccSh via the guide cannula, and modified Ringer's solution (MRS; $150 \mathrm{mM} \mathrm{NaCl}, 3.0 \mathrm{mM} \mathrm{KCl}$, $1.4 \mathrm{mM} \mathrm{CaCl}_{2}$, and $0.8 \mathrm{mM} \mathrm{MgCl} 2$ in $10 \mathrm{mM}$ phosphate buffer at $\mathrm{pH}$ 7.2) was perfused at a constant flow rate of $1.5 \mu \mathrm{L} / \mathrm{min}$ by using a microinjection pump in a microdialysis bowl. Microdialysis samples were collected at 20-min intervals in microcentrifuge tubes and three consecutive samples were assayed using an HPLC procedure with a coulometric detector (Coulochem II; ESA, Bedford, MA, USA) to measure basal DA levels prior to drug administration.

\section{Evaluation of oxidative stress parameters}

The stored NaccSh tissue samples were sonicated (10\%, $w / v)$ in ice-cold $0.1 \mathrm{M}$ phosphate buffer ( $\mathrm{pH} 7.4)$, centrifuged at $700 \times g$ for $10 \mathrm{~min}$ at $4{ }^{\circ} \mathrm{C}$, and the supernatants were collected to measure parameters of oxidative stress. Malondialdehyde (MDA) levels, which are an index of lipid peroxidation, were determined using a thiobarbituric acid method and an MDA kit (Jiancheng Bioengineering Institute, Nanjing, China) [14]. The activities of accumbal antioxidant enzymes, including superoxide dismutase (SOD) and catalase (CAT), were assayed spectrophotometrically using the respective kits from Jiancheng Bioengineering Institute. Protein concentrations in the brain homogenate samples were determined using the bicinchoninic acid protein assay.

\section{Western blot analysis}

Cytosolic and nuclear proteins were extracted from the NaccSh samples for the detection of heme oxygenase 1 (HO-1) and nuclear factor erythroid 2-related factor 2 (Nrf2) using cytoplasmic and nuclear extraction reagents (Beyotime Biotech Inc., Nantong, China). Protein samples were separated using $12 \%$ sodium dodecyl sulfate polyacrylamide gel electrophoresis (SDS-PAGE) and then transferred to polyvinylidene difluoride (PVDF) membranes (Millipore, Bedford, MA, USA). The primary antibodies for the immunoblotting assays included a rabbit polyclonal antibody for Nrf2 (Abcam, Cambridge, UK) and a rabbit polyclonal antibody for HO-1 (Abcam); the secondary antibody was IRDye $800 \mathrm{CW}$ Goat antiRabbit IgG (Li-Cor Bioscience, Lincoln, NE, USA). 


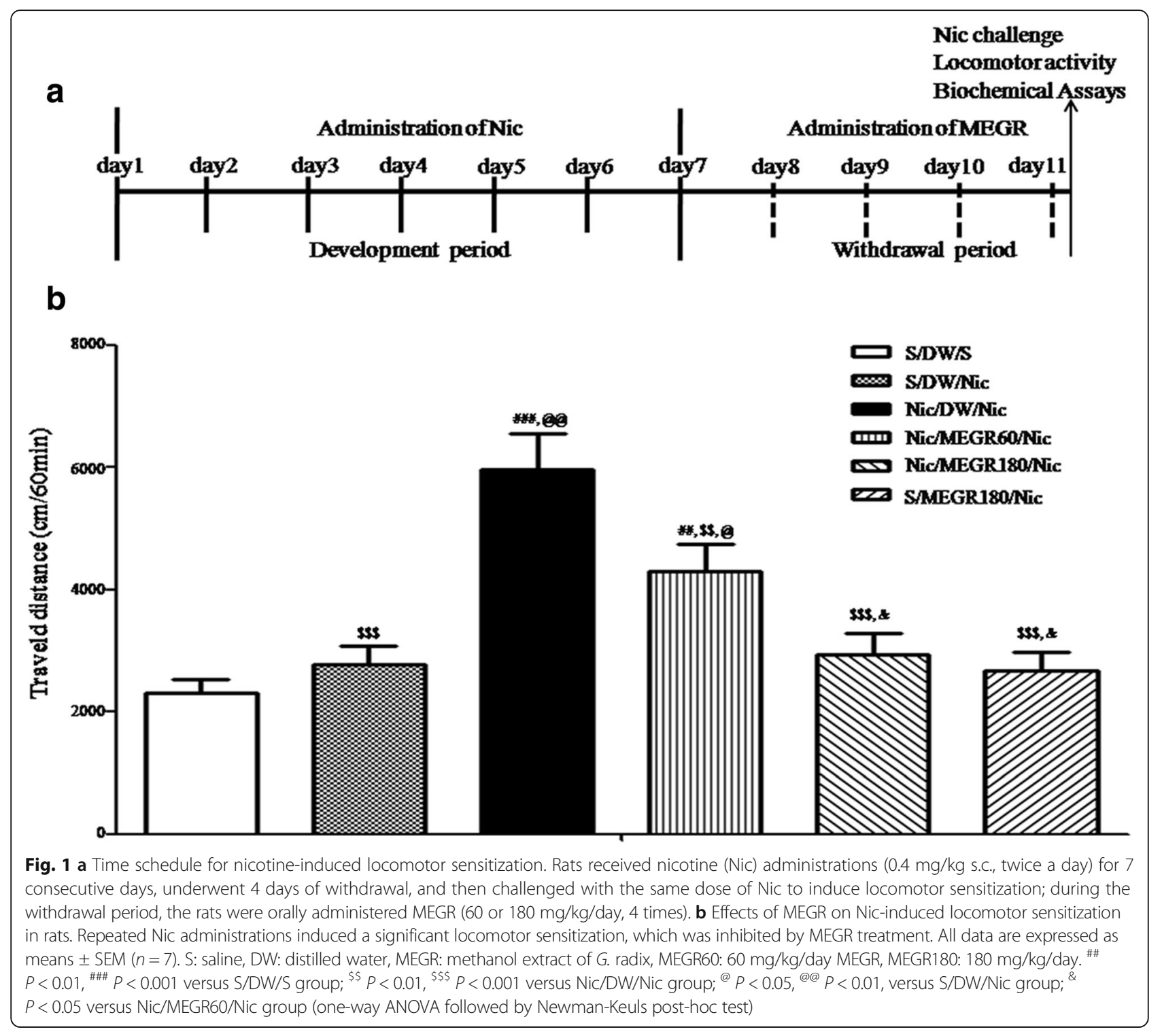

Histone H3 (for nuclear proteins) and $\beta$-actin (for cytoplasmic proteins) were used as loading controls and detected with a rabbit polyclonal antibody (Abcam). The ODYSSEY Infrared Imaging System (Li-Cor Bioscience) was used to detect signals according to the manufacturer's manual.

Intra-NaccSh injection of tert-butyl hydroperoxide

To determine whether the effects of MEGR on nicotine sensitization were mediated by accumbal ROS, the ROS donor tert-butyl hydroperoxide ( $t$-BOOH; $3.0 \mu \mathrm{g} / 200 \mathrm{~nL} /$ side; Sigma-Aldrich) [28] was dissolved in MRS and bilaterally administered into the NaccSh via the injectors (internal cannulae, $17 \mathrm{~mm}$; 28-gauge) using motorized syringe pumps over a period of $60 \mathrm{~s}$ approximately $60 \mathrm{~min}$ after the fourth MEGR treatment. For the intra-NaccSh infusions of $t-\mathrm{BOOH}$, stainless steel guide cannulae
(15 mm; 23-gauge, $2 \mathrm{~mm}$ shorter than the internal cannulae) were bilaterally implanted into the brain using a stereotaxic instrument while the rats were under anesthesia; the cannula tips were placed $2 \mathrm{~mm}$ above the NaccSh so that the injectors exactly targeted the NaccSh. Five minutes after $t$-BOOH administration, the rats were systemically challenged with nicotine and tested in the locomotor testing boxes for $60 \mathrm{~min}$. Immediately after the behavioral test, the rats were decapitated and their brains were removed to verify the guide cannula placement.

\section{Statistical analysis}

All data are expressed as mean \pm standard error of the mean (SEM). The behavioral and biochemical data (including Western blot analyses) were assessed with oneway analysis of variance (ANOVA) tests followed by Newman-Keuls post-hoc tests. The neurochemical data 
(in vivo microdialysis) were assessed with a two-way ANOVA followed by Bonferroni post-hoc tests. All tests were conducted with the commercially available software GraphPad Prism 5.0 (GraphPad Software, San Diego, CA, USA) and $p$ values $<0.05$ were considered to indicate statistical significance. The normality of data was checked and the homogeneity of variances was analyzed by Bartlett's test before each running of ANOVA analyses.

\section{Results}

\section{Effects of MEGR on nicotine-induced Locomotor sensitization}

A nicotine (or saline) challenge was performed on the fourth day after the final daily administration of the repeated nicotine protocol. The RNT rats challenged with nicotine (Nicotine/DW/Nicotine) exhibited a significantly greater increase in locomotor activity than the repeated saline-treated rats (Saline/DW/Saline) and rats that received only a challenge dose of nicotine (Saline/DW/Nicotine) $\left[\mathrm{F}_{(5,36)}=12.46, p<0.0001 ; \quad\right.$ Nicotine $/ \mathrm{DW} /$ Nicotine group $(n=7)$ vs. Saline/DW/Saline group $(n=7), p<0.001$; Nicotine/DW/Nicotine group vs. Saline/DW/Nicotine group $(n=7), p<0.01]$. These findings indicate that RNT induced locomotor sensitization. However, the nicotineinduced behavioral sensitization was attenuated by both doses (60 and $180 \mathrm{mg} / \mathrm{kg} / \mathrm{d}$ ) of MEGR [Nicotine/DW/Nicotine group vs. Nicotine/MEGR60/Nicotine group $(n=7), p$ $<0.01$; Nicotine/DW/Nicotine group vs. Nicotine/ MEGR180/Nicotine group $(n=7), p<0.001]$ in a dosedependent manner (Nicotine/MEGR60/Nicotine group vs. Nicotine/MEGR180/Nicotine group, $p<0.05)$. Locomotor activity was not significantly altered by acute nicotine treatment (Saline/DW/Nicotine group vs. Saline/DW/Saline group, $p>0.05$ ) or treatment with $180 \mathrm{mg} / \mathrm{kg} / \mathrm{d}$ MEGR (Saline/DW/Saline group vs. Saline/MEGR180/Nicotine group, $p>0.05$ ) (Fig. 1b).

\section{Effects of MEGR on nicotine-sensitized increases in Accumbal DA release}

The rats with surgery did not show any significant local infection and physical abnormalities, and there were no significant differences in basal accumbal DA levels among the repeated saline-treated group (Saline/DW/Saline: $3.92 \pm 0.45 \mathrm{nM}, n=5)$, the acute nicotine-treated group (Saline/DW/Nicotine: $4.23 \pm 0.48 \mathrm{nM}, n=5$ ), the RNT group (Nicotine/DW/Nicotine: $3.79 \pm 0.38 \mathrm{nM}, n=$ 6), the repeated nicotine plus $180 \mathrm{mg} / \mathrm{kg} / \mathrm{d}$ MEGR group (Nicotine/MEGR180/Nicotine: $4.01 \pm 0.43 \mathrm{nM}, n=6$ ), and the acute nicotine plus $180 \mathrm{mg} / \mathrm{kg} / \mathrm{d}$ MEGR group (Saline/MEGR180/Nicotine: $4.32 \pm 0.52 \mathrm{nM}, n=5$ ). A two-way ANOVA and post-hoc tests revealed that the acute local infusion of nicotine directly into the NaccSh produced much higher elevations of accumbal DA overflow (relative to baseline) in nicotine-pretreated rats than in saline-pretreated rats $\left[\mathrm{F}_{\text {(treatment } \times \text { time) }}=17.35, p<\right.$ $0.0001 ; \mathrm{F}_{\text {(treatment) }}=58.64, p<0.0001 ; \mathrm{F}_{\text {(time) }}=78.32, p<$ $0.0001]$. Additionally, significant elevations in accumbal DA were seen at $20 \mathrm{~min}$ [Nicotine/DW/Nicotine group (343.24 $\pm 36.47 \%)$ vs. Saline/DW/Saline group, $p<0.001$; Nicotine/ DW/Nicotine group vs. Saline/DW/Nicotine group (201.39 $\pm 21.04 \%), \quad p<0.001], \quad 40 \mathrm{~min}$ [Nicotine/DW/Nicotine group $(700.92 \pm 69.35 \%)$ vs. Saline/DW/Saline group, $p<$ 0.001; Nicotine/DW/Nicotine group vs. Saline/DW/Nicotine group (313.71 $\pm 30.64 \%), p<0.001$ ], and $60 \mathrm{~min}$ [Nicotine/DW/Nicotine group $(286.96 \pm 30.11 \%)$ vs. Saline/DW/ Saline group, $p<0.001$; Nicotine/DW/Nicotine group vs. Saline/DW/Nicotine group (130.90 $\pm 14.31 \%), \quad p<0.001]$ after a nicotine challenge.

However, similar to the locomotor activity results, treatment with MEGR effectively attenuated the sensitized increases in extracellular DA release at $20 \mathrm{~min}$ (Nicotine/ DW/Nicotine group vs. Nicotine/MEGR180/Nicotine group, $p<0.001$ ), $40 \mathrm{~min}$ (Nicotine/DW/Nicotine group vs. Nicotine/MEGR180/Nicotine group, $p<0.001$ ) and $60 \mathrm{~min}$ (Nicotine/DW/Nicotine group vs. Nicotine/MEGR180/ Nicotine group, $p<0.001)$. In contrast, post-hoc tests revealed that, unlike the behavioral data, an acute nicotine infusion into the NaccSh increased accumbal DA release at $20 \mathrm{~min}$ (Saline/DW/Nicotine group vs. Saline/DW/Saline group, $p<0.05$ ) and $40 \mathrm{~min}$ (Saline/DW/Nicotine group vs. Saline/DW/Saline group, $p<0.001$ ); there was a trend for $180 \mathrm{mg} / \mathrm{kg}$ MEGR to inhibit this increase but the difference was not statistically significant (Fig. 2).

\section{Effects of MEGR on antioxidant profiles in the NaccSh}

There were significant increases in accumbal MDA production in the nicotine-sensitized rats (Nicotine/DW/ Nicotine) compared with the repeated saline-treated rats $\left[\mathrm{F}_{(4,18)}=12.31, p<0.0001 ;\right.$ Nicotine $/ \mathrm{DW} /$ Nicotine group $(n=5)$ vs. Saline/DW/Saline group $(n=4), p<0.001]$, indicating nicotine sensitization is associated with increased accumbal oxidative stress; however, treatment with $180 \mathrm{mg} / \mathrm{kg} / \mathrm{d}$ MEGR once a day for $4 \mathrm{~d}$ significantly inhibited these increases [Nicotine/DW/Nicotine group vs. Nicotine/MEGR180/Nicotine group $(n=5), p<0.001$ ] (Fig. 3a). Accumbal SOD and CAT activities in the nicotine-sensitized group were lower than those in the repeated saline-treated group [SOD: $\mathrm{F}_{(4,18)}=4.18, p<$ 0.05 ; Nicotine/DW/Nicotine group $(n=5)$ vs. Saline/ DW/Saline group $(n=4), p<0.05$; CAT: $\mathrm{F}_{(4,18)}=6.27, p$ $<0.01$; Nicotine/DW/Nicotine group $(n=5)$ vs. Saline/ DW/Saline group $(n=4), p<0.01]$. The alterations of SOD and CAT activities were also ameliorated by treatment with MEGR [SOD: Nicotine/DW/Nicotine group vs. Nicotine/MEGR180/Nicotine $(n=5), p<0.05$; CAT: Nicotine/DW/Nicotine group vs. Nicotine/MEGR180/ Nicotine group $(n=5), p<0.01]$ (Fig. $3 b$ and $c)$. 


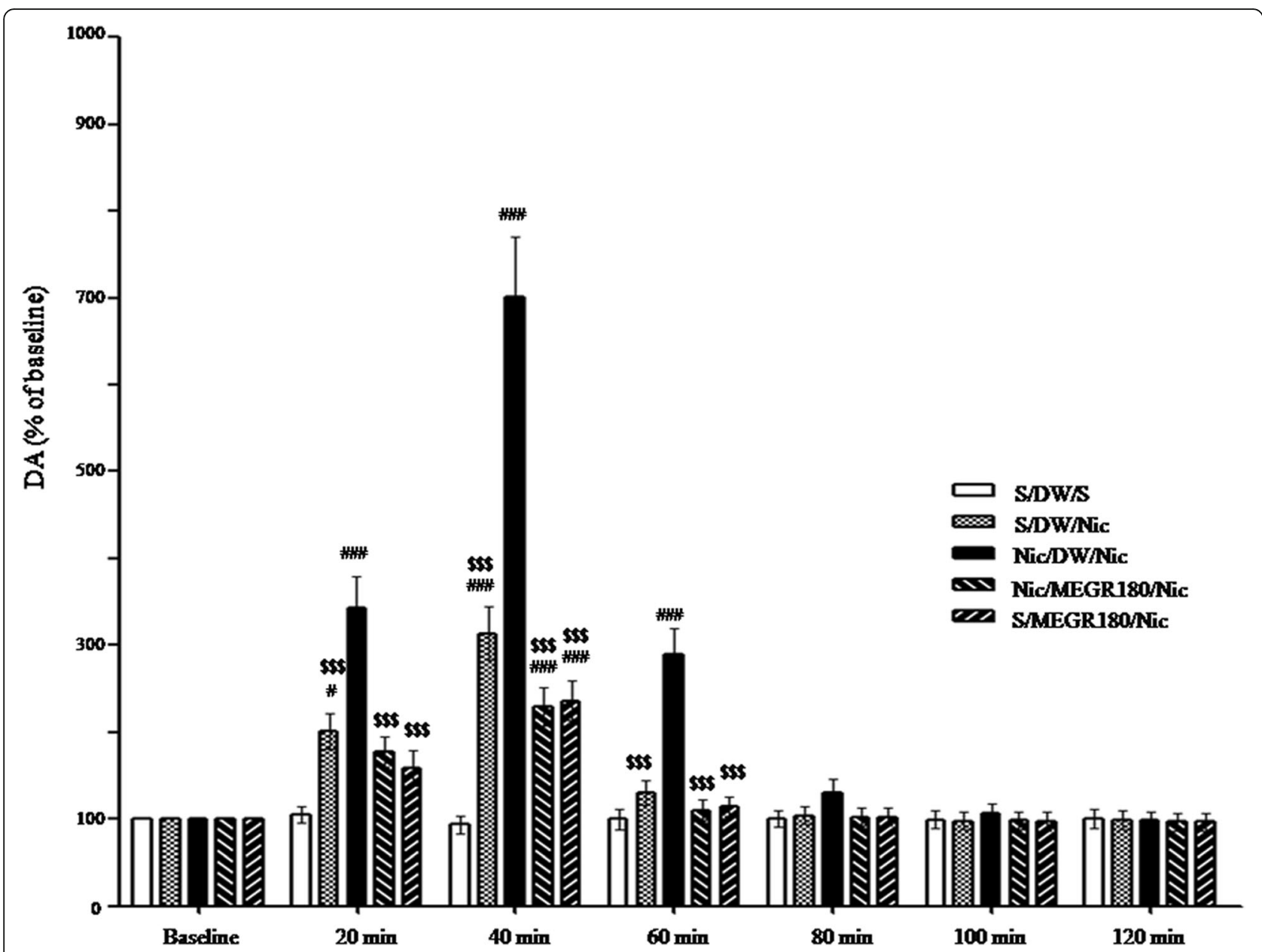

Fig. 2 Effects of MEGR on extracellular DA levels in the NaccSh. On the 4th after the last nicotine (Nic) injection, rats were challenged with a direct intra-NaccSh infusion of $3.0 \mathrm{mM} \mathrm{Nic}$, and during the withdrawal period the rats were treated with MEGR. All data are expressed as means \pm SEM ( $n=5-6)$. Nic: nicotine, S: saline, MEGR: methanol extract of G. radix, MEGR180: $180 \mathrm{mg} / \mathrm{kg} /$ day MEGR once a day for 4 consecutive days. "\# $P<0.05$, \#\#\# $P<0.001$ versus S/DW/S group; ${ }^{\$ \$ \$} P<0.001$ versus Nic/DW/Nic group (two-way ANOVA followed by the Bonferroni post-hoc test)

However, neither acute nicotine treatment nor MEGR administration alone significantly changed the oxidant/ antioxidant state in the NaccSh [MDA, SOD, and CAT: Saline/DW/Nicotine group $(n=5)$ vs. Saline/DW/Saline group, $p>0.05$; Saline/MEGR180/Saline group $(n=4)$ vs. Saline/DW/Saline group, $p>0.05]$.

\section{Effects of MEGR on Nrf2 and HO-1 protein expression in the NaccSh}

The protein expressions of cytoplasmic Nrf2 and HO-1 in the NaccSh exhibited a reduction in the nicotine-sensitized group compared with those in the repeated saline-treated group [Nrf2: $\mathrm{F}_{(3,12)}=4.64, p<0.05$; Nicotine $/ \mathrm{DW} /$ Nicotine group $(n=4)$ vs. Saline/DW/Saline group $(n=4), p<0.05$; HO-1: $\mathrm{F}_{(3,12)}=7.37, p<0.01$; Nicotine/DW/Nicotine group $(n=4)$ vs. Saline/DW/Saline group $(n=4), p<0.01]$. Additionally, the nuclear protein expressions of Nrf2 in the NaccSh were lower in the nicotine-sensitized rats than in the repeated saline-treated rats $\left[\mathrm{F}_{(3,12)}=10.71, p<0.01\right.$;
Nicotine/DW/Nicotine group $(n=4)$ vs. Saline/DW/Saline group $(n=4), p<0.01]$. However, these downregulated protein expressions were reversed by treatment with $180 \mathrm{mg} /$ $\mathrm{kg} / \mathrm{d}$ MEGR once a day for $4 \mathrm{~d}$ during the nicotine withdrawal period [Nrf2 (cytoplasmic): Nicotine/DW/Nicotine group vs. Nicotine/MEGR180/Nicotine group $(n=4), p<$ 0.05; HO-1: Nicotine/DW/Nicotine group vs. Nicotine/ MEGR180/Nicotine group $(n=4), p<0.01$; Nrf2 (nuclear): Nicotine/DW/Nicotine group vs. Nicotine/MEGR180/ Nicotine group $(n=4), p<0.01]$. MEGR treatment alone did not produce significant changes in the cytoplasmic and nuclear protein levels of Nrf-2 or HO-1 [Nrf2 (cytoplasmic), HO-1, and Nrf2 (nuclear): Saline/MEGR180/Saline group $(n=4)$ vs. Saline/DW/Saline group, $p>0.05]$ (Fig. 4).

Effects of intra-NaccSh infusion of $t-\mathrm{BOOH}$ on the antisensitization action of MEGR

Consistent with the locomotor activity findings, RNT sensitized the locomotor response to a challenge dose of 


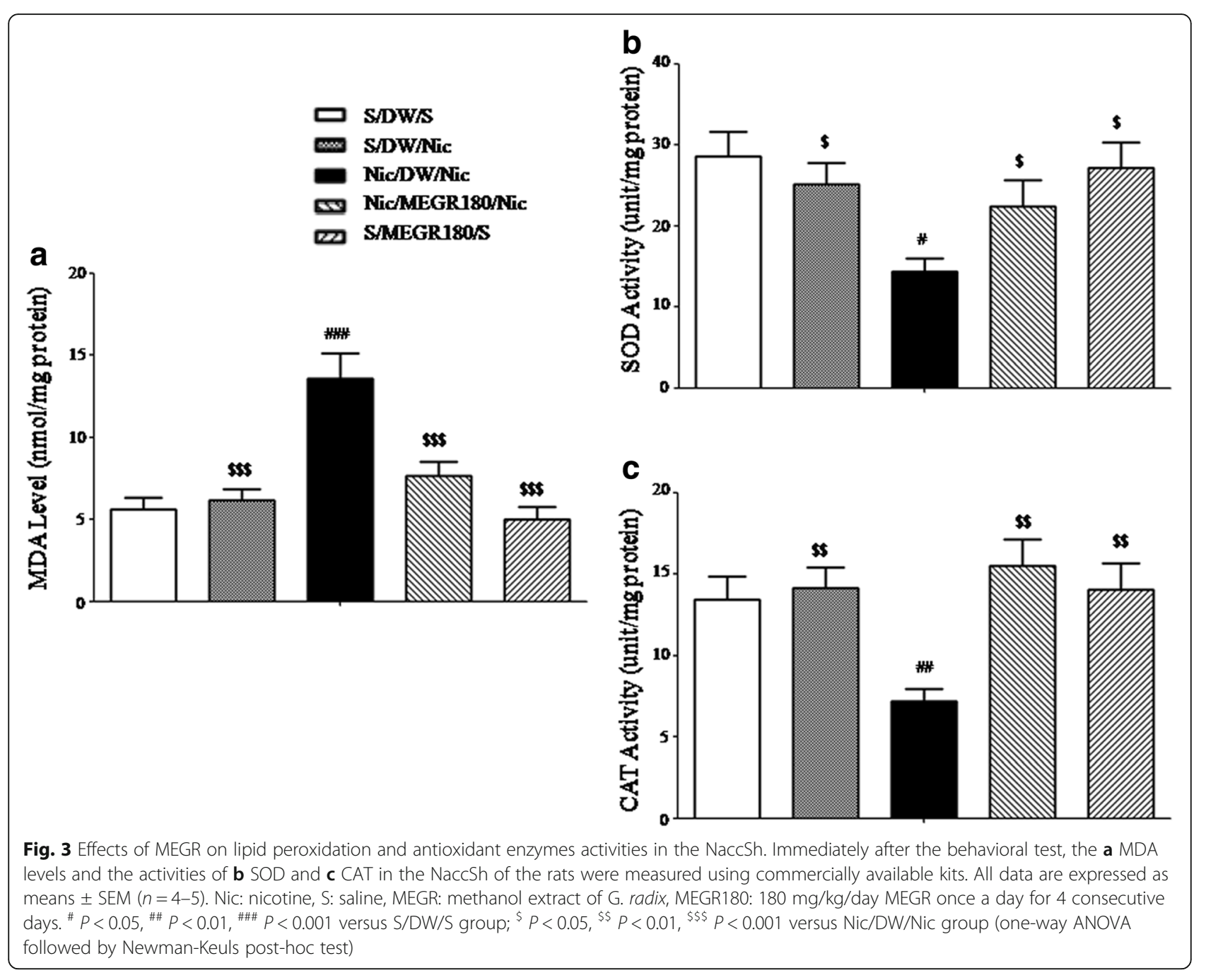

nicotine $\left[\mathrm{F}_{(3,}, 14\right)=8.37, p<0.01 ;$ Nicotine/DW/MRS/ Nicotine group $(n=4)$ vs. Saline/DW/MRS/Saline group $(n=4), p<0.01]$ and $180 \mathrm{mg} / \mathrm{kg} / \mathrm{d}$ MEGR prevented the occurrence of this behavioral sensitization [Nicotine/ DW/MRS/Nicotine group vs. Nicotine/MEGR180/MRS/ Nicotine group $(n=5), p<0.05]$. However, a post-MEGR infusion of $t$ - $\mathrm{BOOH}$ into the NaccSh abolished the ability of MEGR to prevent sensitization [Nicotine/ MEGR180/MRS/Nicotine group vs. Nicotine/MEGR180/ $t$-BOOH/Nicotine group $(n=5), p<0.05$ ] (Fig. 5).

\section{Discussion}

In the present study, RNT-induced locomotor sensitization was dose-dependently inhibited by MEGR (60 or $180 \mathrm{mg}$ / $\mathrm{kg} / \mathrm{d}$ once a day for $4 \mathrm{~d}$ ) such that the $180 \mathrm{mg} / \mathrm{kg} / \mathrm{d}$ dose almost completely abolished the sensitization. Additionally, $180 \mathrm{mg} / \mathrm{kg} / \mathrm{d}$ MEGR blocked the sensitization-induced increase in DA release in the NaccSh. The present study also showed that there was an increase in MDA production, reduced SOD and CAT activities, and decreases in the expression of $\mathrm{Nrf} 2$ and $\mathrm{HO}-1$ in the nicotine-sensitized $\mathrm{NaccSh}$; these changes were all ameliorated by $180 \mathrm{mg} /$ $\mathrm{kg} / \mathrm{d}$ MEGR. These results suggest that MEGR exerted inhibitory effects on nicotine-induced locomotor activity and accumbal dopaminergic sensitization via its antioxidant action.

In the present study, the repeated systemic administration of nicotine induced behavioral sensitization as evidenced by the increased distance traveled by nicotinepretreated rats than by saline control rats when reexposed to the same dose of nicotine $[6,26]$. This behavioral sensitization was blocked by both doses of MEGR (60 and $180 \mathrm{mg} / \mathrm{kg} / \mathrm{d}$ ), which is consistent with previous findings from our research group showing that these same doses of MEGR attenuated repeated methamphetamineinduced locomotor sensitization [21]. These results indicate that MEGR has an inhibitory effect on nicotineinduced locomotor sensitization. Locomotor sensitization is a behavioral phenotype associated with a sensitized mesoaccumbal dopaminergic response, which is also 


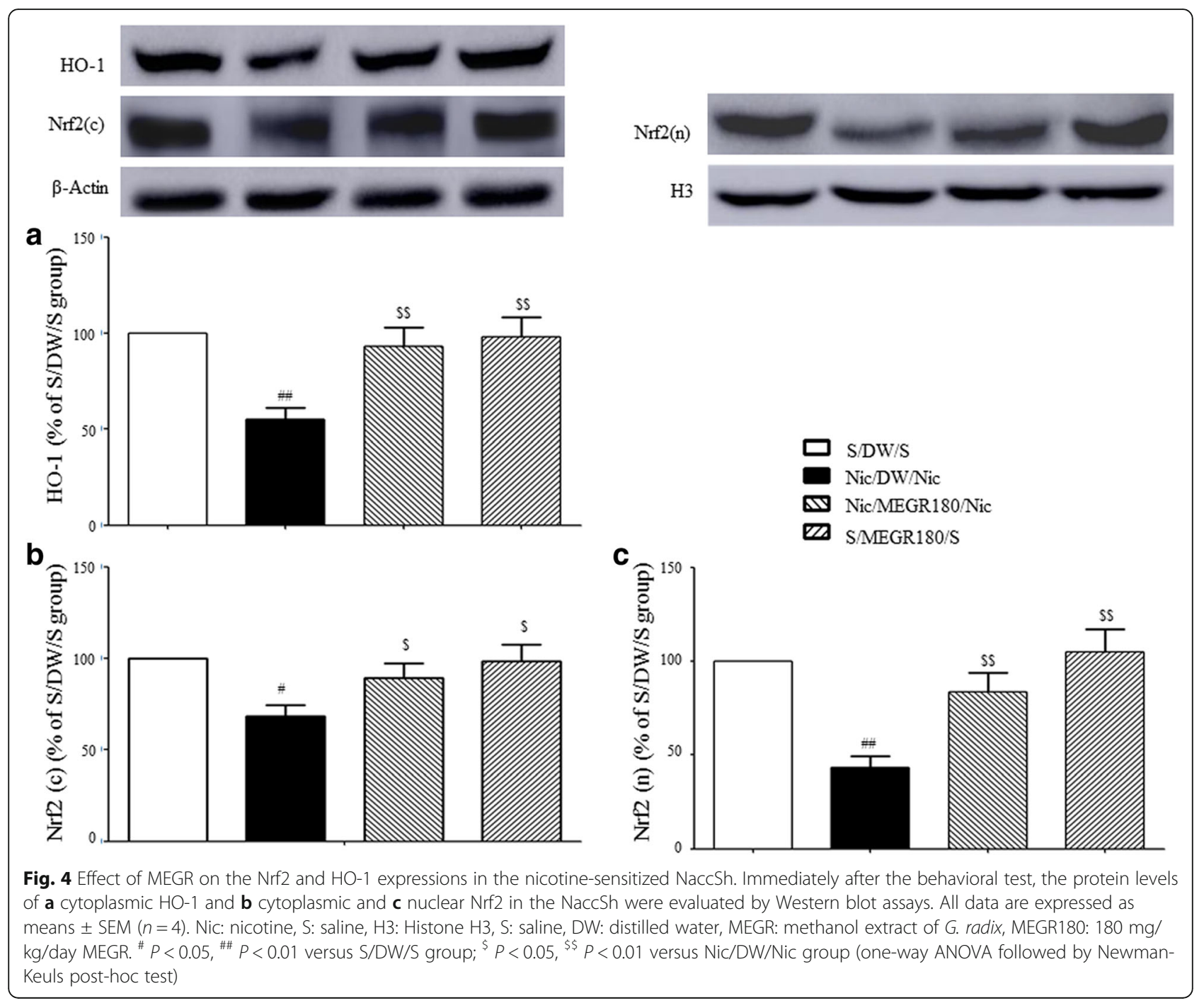

supported by previous findings from our group [6, 26]. In the present study, a challenge infusion of nicotine into the NaccSh elicited significantly greater accumbal DA release in nicotine-pretreated rats than in saline control rats. However, this increase was effectively abolished by $180 \mathrm{mg} / \mathrm{kg} / \mathrm{d}$ MEGR. These results provide neurochemical support for the inhibitory effects of MEGR on RNTinduced behavioral sensitization and imply that the behavioral effects are mediated by the ability of MEGR to mask the sensitized accumbal DA response.

Nicotine binds to nicotinic acetylcholine receptors (nAchRs) to induce neurotransmitter release, including accumbal DA, in the brain [29]. This action is physiologically designated as neuronal signal transmission as well as biochemically denoted by an altered cellular energy state coupled with the production of ROS [30]. RNT alters activity within the tegmental-accumbal dopaminergic system that underlies behavioral and accumbal dopaminergic sensitization, which is biochemically sustained by heightened accumbal ROS levels. RNT increases the production of lipid hydroperoxides in the cerebral cortex and hippocampus of rats [31]. RNT-induced behavioral sensitization and conditioned place preference in mice occur in conjunction with the dysregulation of accumbal membrane metabolism and an increase in hippocampal MDA production [32, 33]. Our group has previously reported that rats self-administering cocaine and methamphetamine show an enhanced production of accumbal ROS, which is associated with sensitized accumbal DA release $[9,10]$. The present study found RNT-induced elevation in MDA levels and decreases in SOD and CAT activities in the NaccSh, which supports the finding that repeated psychostimulant treatment increases accumbal oxidative stress. The present study also found that $180 \mathrm{mg} / \mathrm{kg} / \mathrm{d}$ MEGR prevented increases in accumbal oxidative stress and that the post-MEGR administration of $t-\mathrm{BOOH}$ abolished the inhibitory effects of MEGR on nicotine-induced locomotor sensitization. These results indicate that MEGR 


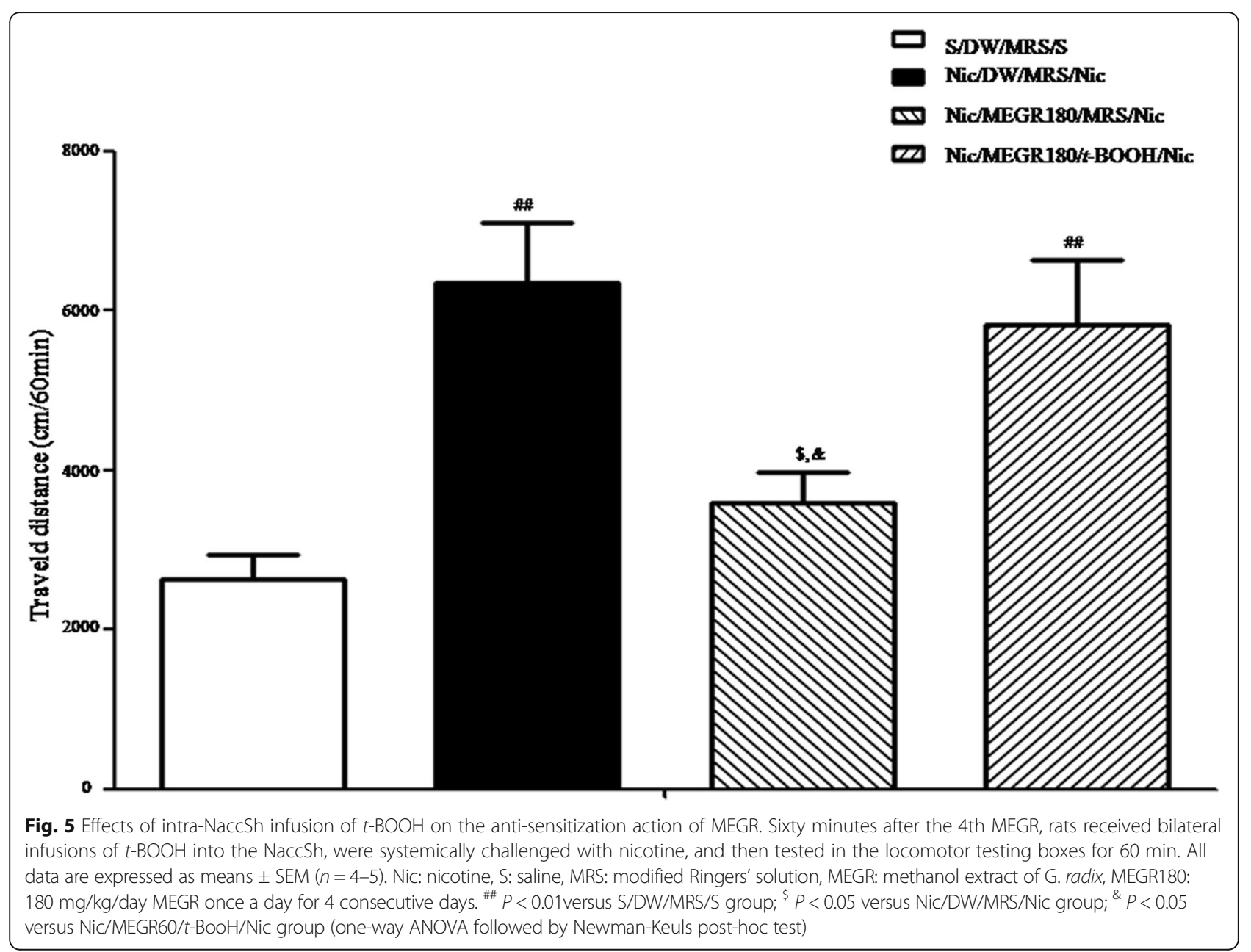

blocks RNT-induced sensitization by counteracting increases in accumbal oxidative stress.

Eukaryotic cells have a fine-tuned oxidant/antioxidant system that maintains normal cellular functioning. Nrf2 is a basic leucine zipper transcription factor that plays a central role in the antioxidant process. Under normal conditions, it resides in the cytoplasm and is quickly degraded by a cluster of proteins, including Keap 1 and Cullin3. However, once stressful events occur, Nrf2 is disassociated from Keap1 and translocated into the nucleus to bind to DNA promoters where it eventually initiates the transcription of several antioxidant and phase II detoxifying enzymes, including HO-1 [34], thus protecting cells against injury.

In the present study, RNT reduced Nrf2 protein expression in both the cytoplasmic and nuclear fractions of the NaccSh, which is consistent with studies showing that beta-amyloid-induced stress decreased hippocampal Nrf2 expression [35] and that traumatic brain injury disrupted the nuclear translocation of Nrf2 in the cerebral cortex [36]. The present study also found decreased HO-1 expression in the nicotine-sensitized NaccSh.
Taken together, these results indicate that RNT elicits dysregulation in the Nrf2-HO-1 pathway and that the disturbance in cellular antioxidant function underlies the enhancement of accumbal oxidative stress which, in turn, further mediates RNT-induced behavioral and neurochemical sensitization. On the other hand, the present study also showed that $180 \mathrm{mg} / \mathrm{kg} / \mathrm{d}$ MEGR markedly increased the expression of Nrf2 and HO-1 in the nicotine-sensitized NaccSh but spared it in the nicotine-free NaccSh. Taken together, these findings suggest that the effects of MEGR on RNT-induced sensitization and accumbal oxidative stress are due to improvements in altered cellular antioxidant systems, such as the Nrf2-HO-1 pathway.

\section{Conclusions}

In the present study, 60 and $180 \mathrm{mg} / \mathrm{kg} / \mathrm{d}$ MEGR dosedependently suppressed RNT-induced locomotor sensitization and $180 \mathrm{mg} / \mathrm{kg} / \mathrm{d}$ MEGR reduced sensitized accumbal DA release. Additionally, MEGR attenuated RNT-induced increases in MDA levels and decreases in SOD and CAT activities in the NaccSh and rescued 
downregulated function in the Nrf2-HO-1 pathway. These data suggest that $G$. radix can block RNT-induced behavioral and neurochemical sensitization by counteracting accumbal oxidative stress, and further provide the possibility that $G$. radix can be an important herbal source to develop new agents to aid smoking cessation.

\section{Abbreviations}

CAT: Catalase; DA: Dopamine; DW: Distilled water; G. radix: Glycyrrhizae radix; HO-1: Heme oxygenase 1; HPLC: High-performance liquid chromatography; MDA: Malondialdehyde; MEGR: Methanol extract from G. radix; MEGR60 (180): 60 (180) mg/kg/d MEGR; MRS: Modified Ringer's solution;

Nacc: Nucleus accumbens; NaccSh: Nacc shell; Nrf2: Nuclear factor erythroid 2-related factor 2; RNT: Repeated nicotine treatment; ROS: Reactive oxygen species; SOD: Superoxide dismutase; $t-\mathrm{BOOH}$ : tert-butyl hydroperoxide

\section{Acknowledgements}

This study was supported by the Science Research Foundation of Qiqihar Medical University, China (QY2016GJ-01) and the National Research Foundation of Korea (NRF) funded by the Korean government (MSIP) (no. 2012 R1A5A2A42671316).

\section{Funding}

1. The Science Research Foundation of Qiqihar Medical University, China (QY2016GJ-01);

2. The National Research Foundation of Korea (NRF) funded by the Korean government (MSIP) (no. 2012 R1A5A2A42671316).

\section{Availability of data and materials}

The data collected in the present study are properly analyzed and summarized in Methods section and Results section, and are available from the corresponding author upon reasonable request. All materials used in this study are properly included in Methods section.

\section{Authors' contributions}

RZ and SCK designed the research; ZZ, HL, YW (YiYan WU), LL, YJ, YF and $\mathrm{BHL}$ performed the research, SCK and IJC prepared the methanol extract from G. radix, YW(YuHua Wang) and CHY analyzed data, RZ, SCK and CWL wrote the paper. All authors read and approved the final content of the manuscript.

\section{Ethics approval}

All animal experiments were approved by the Animal Ethical Care Committee of Qiqihar Medical University (Number: QMU-AECC-2015-26) and conducted in accordance with the guidelines for the care and use of laboratory animals at Qiqihar Medical University.

\section{Consent for publication}

$$
\text { Not applicable. }
$$

\section{Competing interests}

The authors declare that they have no competing interests.

\section{Publisher's Note}

Springer Nature remains neutral with regard to jurisdictional claims in published maps and institutional affiliations.

\section{Author details \\ ${ }^{1}$ School of Mental Health, Qiqihar Medical University, 333 Bukuibei Street, Jianhua District, Qiqihar 161006, China. ${ }^{2}$ College of Korean Medicine, Daegu Haany University, Gyeongsan 38610, Republic of Korea. ${ }^{3}$ Department of Pharmacology, Mudanjiang Medical University, Mudanjiang 157011, China.}

Received: 21 July 2017 Accepted: 10 November 2017 Published online: 16 November 2017

\section{References}

1. Ladapo JA, Prochaska JJ. Paying smokers to quit: does it work? Should we do it? J Am Coll Cardiol. 2016;68(8):786-8.
2. Reid RD, Pritchard G, Walker K, Aitken D, Mullen KA, Pipe AL. Managing smoking cessation. CMAJ. 2016;188(17-18):E484-92.

3. Eggan BL, McCallum SE. 18-Methoxycoronaridine acts in the medial habenula to attenuate behavioral and neurochemicalsensitization to nicotine. Behav Brain Res. 2016;307:186-93.

4. Gomez AM, Sun WL, Midde NM, Harrod SB, Zhu J. Effects of environmental enrichment on ERK1/2 phosphorylation in the rat prefrontal cortex following nicotine-induced sensitization or nicotine self-administration. Eur J Neurosci. 2015;41(1):109-19.

5. Corrigall WA, Coen KM, Adamson KL. Self-administered nicotine activates the mesolimbic dopamine system through the ventral tegmental area. Brain Res. 1994;653(1-2):278-84.

6. Shim I, Javaid JI, Wirtshafter D, Jang SY, Shin KH, Lee HJ, Chung YC, Chun BG. Nicotine-induced behavioral sensitization is associated with extracellular dopamine release and expression of c-Fos in the striatum and nucleus accumbens of the rat. Behav Brain Res. 2001;121(1-2):137-47.

7. Uys JD, Knackstedt L, Hurt P, Tew KD, Manevich Y, Hutchens S, Townsend DM, Kalivas PW. Cocaine-induced adaptations in cellular redox balance contributes to enduring behavioral plasticity. Neuropsychopharmacology. 2011;36:2551-60.

8. Biala G, Pekala K, Boguszewska-Czubara A, Michalak A, Kruk-Slomka M, Grot K, Budzynska B. Behavioral and biochemical impact of chronic unpredictable mild stress on the Acquisition of Nicotine Conditioned Place Preference in rats. Mol Neurobiol. 2017; doi: 10.1007/s12035-017-0585-4.

9. Jang EY, Ryu YH, Lee BH, Chang SC, Yeo MJ, Kim SH, Folsom RJ, Schilaty ND, Kim KJ, Yang CH, Steffensen SC, Kim HY. Involvement of reactive oxygen species in cocaine-taking behaviors in rats. Addict Biol. 2015;20(4):663-75.

10. Jang EY, Yang CH, Hedges DM, Kim SP, Lee JY, Ekins TG, Garcia BT, Kim HY, Nelson AC, Kim NJ, Steffensen SC. The role of reactive oxygen species in methamphetamine self-administration and dopamine release in the nucleus accumbens. Addict Biol. 2016; doi: 10.1111/adb.12419.

11. Numa R, Kohen R, Poltyrev T, Yaka R. Tempol diminishes cocaine-induced oxidative damage and attenuates the development and expression of behavioral sensitization. Neuroscience. 2008;155:649-58.

12. Wang ZY, Nixon DW. Licorice and cancer. Nutr Cancer. 2001;39(1):1-11.

13. Kamei J, Nakamura R, Ichiki H, Kubo M. Antitussive principles of Glycyrrhizae radix, a main component of the Kampo preparations Bakumondo-to (Maimen-dong-tang). Eur J Pharmacol. 2003;469(1-3):159-63.

14. Zhao Z, Park SM, Guan L, Wu Y, Lee JR, Kim SC, Kim YW, Zhao R. Isoliquiritigenin attenuates oxidative hepatic damage induced by carbon tetrachloride with or without buthionine sulfoximine. Chem Biol Interact. 2015;225:13-20.

15. Kim SC, Park SJ, Lee JR, Seo JC, Yang CH, Byun SH. Cytoprotective activity of Glycyrrhizae radix extract against Arsenite-induced Cytotoxicity. Evid Based Complement Alternat Med. 2008;5(2):165-71.

16. Lee HK, Yang EJ, Kim JY, Song KS, Seong YH. Inhibitory effects of Glycyrrhizae radix and its active component, isoliquiritigenin, on A $\beta$ (25-35)induced neurotoxicity in cultured rat cortical neurons. Arch Pharm Res. 2012;35(5):897-904.

17. Zhang K, Wang F, Yang JY, Wang LJ, Pang HH, Su GY, Ma J, Song SJ, Xiong ZL, Wu CF. Analysis of main constituents and mechanisms underlying antidepressant-like effects of Xiaochaihutang in mice. J Ethnopharmacol. 2015;175:48-57.

18. Park HJ, Shim HS, Kim H, Kim KS, Lee H, Hahm DH, Shim I. Effects of Glycyrrhizae radix on repeated restraint stress-induced Neurochemical and Behavioral responses. Korean J Physiol Pharmacol. 2010;14(6):371-6.

19. Jang EY, Choe ES, Hwang M, Kim SC, Lee JR, Kim SG, Jeon JP, Buono RJ, Yang $\mathrm{CH}$. Isoliquiritigenin suppresses cocaine-induced extracellular dopamine release in rat brain through $\mathrm{GABA}(\mathrm{B})$ receptor. Eur J Pharmacol. 2008;587(1-3):124-8.

20. Zhao Z, Wang Y, Lin F, Fu H, Zhou F, Chang S, Han NR, Jung DH, Yang CH, Kim SC, Zhao R. Methanol extract from radix of Glycyrrhizae uralensis attenuate methamphetamine-induced hyperlocomotor activity. Herb Formula Sci. 2014;20(1):105-12.

21. Zhao Z, Kim YW, Yang Y, Zhang J, Jung JY, Chang S, Cho IJ, Zhou F, Zhao J, Lee $\mathrm{BH}$, Yang $\mathrm{CH}$, Kim SC, Zhao R. Glycyrrhizae radix methanol extract attenuates methamphetamine-induced locomotor sensitization and conditioned place preference. Evid Based Complement Alternat Med. 2014; 2014:152063. doi: 10.1155/2014/152063.

22. Lee MJ, Yang CH, Jeon JP, Hwang M. Protective effects of isoliquiritigenin against methamphetamine-induced neurotoxicity in mice. J Pharmacol Sci. 2009;111(2):216-20. 
23. Nyberg F. Structural plasticity of the brain to psychostimulant use. Neuropharmacology. 2014;87:115-24.

24. Kawakami Z, Ikarashi $Y$, Kase $Y$. Isoliquiritigenin is a novel NMDA receptor antagonist in kampo medicine yokukansan. Cell Mol Neurobiol. 2011;31(8): 1203-12.

25. Zeng J, Chen Y, Ding R, Feng L, Fu Z, Yang S, Deng X, Xie Z, Zheng S. Isoliquiritigenin alleviates early brain injury after experimental intracerebral hemorrhage via suppressing ROS- and/or NF-KB-mediated NLRP3 inflammasome activation by promoting Nrf2 antioxidant pathway. J Neuroinflammation. 2017;14(1):119.

26. Zhao RJ, Koo BS, Kim GW, Jang EY, Lee JR, Kim MR, Kim SC, Kwon YK, Kim KJ, Huh TL, Kim DH, Shim I, Yang CH. The essential oil from Angelica Gigas NAKAI suppresses nicotine sensitization. Biol Pharm Bull. 2005;28(12):2323-6.

27. Paxinos G, Watson C. The rat brain in Stereotaxic coordinates. San Diego: Academic Press; 1988.

28. Qi C, Wang X, Ge F, Li Y, Shen F, Wang J, Cui C. mGluR5 in the nucleus accumbens shell regulates morphine-assocated contextual memory through reactive oxygen species signaling. Addict Biol. 2015;20(5):927-40.

29. Nocente R, Vitali M, Balducci G, Enea D, Kranzler HR, Ceccanti M. Varenicline and neuronal nicotinic acetylcholine receptors: a new approach to the treatment of co-occurring alcohol and nicotine addiction? Am J Addict. 2013:22(5):453-9.

30. Kovacic P. Unifying mechanism for addiction and toxicity of abused drugs with application to dopamine and glutamate mediators: electron transfer and reactive oxygen species. Med Hypotheses. 2005;65(1):90-6.

31. Toledano A, Alvarez Ml, Toledano-Diaz A. Diversity and variability of the effects of nicotine on different cortical regions of the brain-therapeutic and toxicological implications. Cent Nerv Syst Agents Med Chem. 2010;10(3): 180-206.

32. Budzynska B, Boguszewska-Czubara A, Kruk-Slomka M, Kurzepa J, Biala G. Mephedrone and nicotine: oxidative stress and behavioral interactions in animal models. Neurochem Res. 2015;40(5):1083-93.

33. Li H, Chen B, Shao X, Hu Z, Deng Y, Zhu R, Li Y, Zhang B, Hou J, Du C, Zhao Q, Fu D, Bu Q, Zhao Y, Cen X. 'H-nuclear magnetic resonance-based metabolomic analysis of brain in mice with nicotine treatment. BMC Neurosci. 2014;15:32.

34. Done AJ, Traustadóttir T. Nrf2 mediates redox adaptations to exercise. Redox Biol. 2016;10:191-9.

35. Kim J, Cho CH, Hahn HG, Choi SY, Cho SW. Neuroprotective effects of Nadamantyl-4-methylthiazol-2-amine against amyloid $\beta$-induced oxidative stress in mouse hippocampus. Brain Res Bull. 2017;128:22-8.

36. Li X, Wang H, Gao Y, Li L, Tang C, Wen G, Zhou Y, Zhou M, Mao L, Fan Y. Protective effects of Quercetin on mitochondrial biogenesis in experimental traumatic brain injury via the Nrf2 Signaling pathway. PLoS One. 2016; 11(10):e0164237

\section{Submit your next manuscript to BioMed Central and we will help you at every step:}

- We accept pre-submission inquiries

- Our selector tool helps you to find the most relevant journal

- We provide round the clock customer support

- Convenient online submission

- Thorough peer review

- Inclusion in PubMed and all major indexing services

- Maximum visibility for your research

Submit your manuscript at www.biomedcentral.com/submit
Biomed Central 